\title{
DETERMINATION OF THE CONDITION OF THE OPTIMAL CONTROL OF SURFACE MOISTURE OF SOILS AS A MAJOR FACTOR FOR PRODUCING ENVIRONMENTALLY FRIENDLY AGRICULTURAL PRODUCTS
}

\author{
Prof. akademcian RAS B.H.Aliyev; prof.RAE Z.H.Aliev
}

E_mail: Volad_5@mail.ru

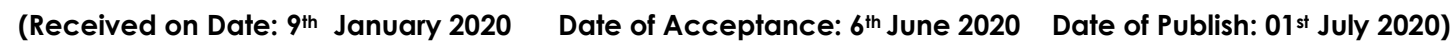

\section{ABSTRACT}

The current information on moisture and the temperature of the ground in managerial system by production to agricultural product necessary, in the first place, for taking the operative decisions at development ecological clean technology irrigation under growing agricultural cultures to achieve the maximum harvest.

Key words: aerospace methods, COW, moisture, moisture test, arable, soil, ecology, vaferhumidity, drill, graduation, tool, etc. 


\section{INTRODUCTION}

The increasing growing of the population in republic puts the problems, provision their provisions including product agricultural production.

The known that in Azerbaijan, as from 50yh years past centuries in broad scale is realized irrigation - ameliorative construction, which has got the big range after 70-h years. The year for year was increased rates water economy construction.

As a result this:

- irrigated area increased from 1200 thou. ha before 1400 thou. ha;

- total water withdrew increased on 9,8 mln. m3;

- an area, engulfed collector-drainage by network bringing before 400 thou. ha; lopening collector-drainage network 273,0; closing - 115,0 thou. ha; and vertical drainage before 5 thou. ha).

Noticeably increased the harvest of the agricultural cultures, So for instance pat reached 30,8 c/ha; corn -24,7 c/ha and vegetables $-204 \mathrm{c} / \mathrm{ha}$.

Alongside with reached success in water economy construction, in prospect follows to execute the following ameliorative of the action:

- a reconstruction to irrigation network in separate region of the republic;

- an improvement ameliorative conditions of the lands:

- increasing water provides agricultural cultures;

- a planning the lands;

- a construction small pools;

- a development and broad introducing the systems water protect few intensive of the technology and technologies of the irrigation agricultural cultures;

-a mastering (for necessities agricultural production) of the additional areas, by introduction is blazed-irrigated husbandries;

- a realization to mechanizations and automations of the process of the irrigation agricultural cultures and etc.

- an using the progressive methods and technical facilities of the study of the condition of ground and receptions express-measurements agro ameliorative parameter and others (including aerospace measurement)

- an introduction to enabling technologies on production of the ecological clean product and increasing to productivities

Follows to note that efficiency of the use progressive water protect technologies of the irrigation and the further his developments in republic consists in that to develop and introduce in production the most latest achievements national and foreign science, technology and leading experience in purpose of the provision high rate production and increasing to their cost-performance, all measured of the growing to capacity of the lab our, culture level increasing of the husbandry, best use the production assets, improvements quality irrigation of the work and increasing to reliability of the applicable technology.

In connection with growth technique contamination surrounding ambiences, appears the problem of the reception to ecological clean product agricultural production.

Full information needs for this about condition of ground, about their п^одородии, about intensities of the contamination of ground chemical element, pesticides, radio nuclides etc., 
as well as dependency of this contamination from hydro meteorology of the conditions.

Since ground-main natural facility production provisions.

Moisture and temperature of the ground one of the main physical characteristic grounds, which define their fertility.

Without presence necessary (required) amount water in ground and corresponding to temperature agricultural cultures do not sprout in general.

Moisture of ground influences upon dissolve, displacement and efficiency organic and mineral fertilizers, on degree of the contamination of ground pesticides and the other product techniques origins, on that, on how much agricultural plants will adopt bad for health of the people chemists. The current information on moisture and the temperature of the ground in managerial system by production to agricultural product necessary, in the first place, for taking the operative decisions at development ecological clean technology irrigation under growing agricultural cultures to achieve the maximum harvest.

There are in view of technological decisions (so for instance, agro techniques of the action) in questions main and before sowing of the processing of ground, practicability and periods of the sowing agricultural cultures, rates and periods irrigation and contributing the mineral fertilizers etc.

However management body, science, production have far from full operative information on moisture of ground through lack of the necessary methods and technical facilities of their reception in branches agricultural production country.

Besides, most exactly and operative possible was get information on moisture and the temperature of the ground by means of artificial companion, but this information is limited only by surface of the land or several centimeters upper layer ground.

Here in after herewith got from cosmos data, necessary to recognize.

But this signifies necessary graduate sensors artificial companion land.

That this realizes, necessary to have a reliable overland express-methods and technical facilities of the reception to information on moisture and temperature of the ground.

Such methods of the facility must provide the reception to information in deep cut (though, on depth lied root system of the plants) and area agricultural fields).

The most known method of the reception given about moisture of ground from her surfaces before depth 100-150 sm, layer through each $10 \mathrm{sm}$, is thermostat-weight (TW) method.

He presents the possibility to get given about moisture of ground for the whole depth lied cortex plants, which puts his untouchable with aerospace methods if the question is objective information in what, that determined point agricultural fields.

But if the question is relative surface moistening of the whole field, that here already TW method, as well as any other overland way, cannot compete c But if the question is relative surface moistening of the whole field, that here already TW.

The purpose of the removal aforesaid defect in 2001 given design was modified on VPG-4C which has exceeded TWmethod on all factors, in that power and 
on accuracy given about moisture of ground on separate agriculture field (the land). The standard deviation herewith from given thermostat-4 weight of the method for VPG-4C formed 1,5\%.

This on $0,5 \%$ more exactly, than shows VNP-1 and on 1,0\% more exactly VPG-1.

But obtain this manages due to the fact that in process of the measurement to moisture applying new way measurements to electric capacity and moisture.

Relies on that main way of the reception most external evidence about moisture of ground, in the opinion of many researchers, is TW-method.

However herewith, as a rule, do not take into account that probe methods does not display on measured space field level to moisture therefore that moisture of ground is defined not 10-refer to layer of the whole field, occupied concrete agriculture by culture, but in that sample of ground only, which is selected for analysis in 40-50 gr aluminum bank.

Moisture measures in ditto time instrument VPG-4C in volume of ground, which reminds the form of the cylinder, height $10 \mathrm{sm}$ and diameter $60 \mathrm{sm}$, but this was test by weight, beside $40-50 \mathrm{~kg}$ dry mass. It must be assumed that similar defect photo optics moisture measure soil sample and portable IK- moisture measure IRMM-106. To use both instruments for measurement of moisture necessary to take out the tests by soil Boer.

More over these tests of the insufficient volume.

The advantage VPG-4C is concluded and hedgehog in that that her possible also to measure and the temperature of the ground. The particularity of this method is concluded, that that he more sensitive to capacitive forming complex electro conductivity ground, than all known hitherto her predecessors.

It is necessary to note, at present in facilities and institutions of the agricultural profile to lead stale work, directed on collection, processing, conservation and transmission to information on surrounding ambience, condition of ground, plants etc. that requires the material expenses and big manual labour, which brings about essential fascination of the cost to information.

The broad automation of the agricultural work with using modern computer technology has allowed not only amounts of the manual works of time, but reduce the cost to information and that particularities it is important, essential image to perfect her quality and operation.

The truth exist and foreign remote points as production of the finish company "Vaysal", automated weather station general Israel-American production and automated meteorological station NPO "Agate" and others follows to note that nor one of considered systems not completely answers the problem and requirements agro meteorology since does not offer the real sensor for measurement of moisture of ground, without which automation of the measurements is an few effective.

The necessary development and introduction automated measuring systems for cathedral, processing, keeping and transmissions to information on condition of ground, connected with decision of the complex of the problems. Here the most important is a measurement speaker's moisture spare in ground. 
But without decision of the problems of the creation exact measure sensor to moisture of ground all deal to automations measurement other parameter of ground in agro amelioration, as particularly important for conditions of the mountain husbandry in region really turn out to be the few effective.

At presence sensor to moisture of ground already become important also sensors of the temperature, density, acidity and others parameter of ground.

The broad automation of the measurements agro ameliorative parameter of ground can precede creation and introduction regional remote agro meteorology informationreference points, which allow to feel the sensors and measuring systems, process the material for the reason reduction of labor content and cost to source information about condition of ground.

It must be assumed that here in after state of working these points, possible easy automates with infrastructure observant meteorological zonal station.

As a result of searching for of the decisions of the problems became known that NPO "Selta", NPO "Agate", ANAKA AN ARE, have comprised of kit of its system project meter to moisture of ground with using the perspective method infrared (IK) spectroscopy.

However if take even fine film of ground by thickness several microns, that she is absolutely impervious for IK- rays.

Possible using the approach, founded on reflection IK- rays, but this approach on given time not it is enough not explored.

Really method IK spectroscopy possible to use not only for determination of the contents of the miscellaneous material in composition mixture, but also in all events he and for determination other necessary parameter, also can is used in variant of the drive IK- rays through under investigation object.

Besides method IK- spectroscopy, pertains to more expensive and suitable to laboratory studies, rather than for development field express-instrument and automated remote device of the measurement to moisture of ground.

However on this cause, is considered reasonable acceptance in attention of the own opinions of the row scientist. The results of the analysis of these studies prove that no united thought (the offers) comparatively principle of the building automated measuring systems in agro meteorology not only, but also hydro meteorology as a whole.

The most making, for methods and facilities of the automatic checking hydro ameliorative parameter, on our glance is a finish company "Vaysal", which offers the consumer certain automatic system for observation for condition many element weathers on the base specialized (meteorological) of the computer.

However system of the company "Vaysal" is "locked" i.e. without special meteorological computer sensors of the company can be not used.

Besides, for the reason decisions of the similar problems, UKRNIGMI was designed automatic system of the checking parameter ground, which on many sign broader possibilities dominates the similar development of the company "Vaysal".

Since proposed UKRNIGMI system is open for any sensor and can be straight connected to computer network through standard relationship without additional connecting device. 
The system will allow simultaneously to remove and send given from big amount sensor on distance before $10 \mathrm{~km}$ by means of cable and before $100 \mathrm{~km}$ and more with use the facility radio system.

Besides graduation parameters sensor is provided bring in memory PC beforehand for constant keeping and remove given with 200-300 sensors for 1 second, rather than enter them before measurement by means of each separate sensor, what the company "Veysal" offers.

The author's offer, for completing automated agro meteorology of the point sensors and measuring device (the own production) with the following parameter:

- moisture of the air (range 25-100\%, at temperature from -4 before $+500 \mathrm{C}$ )

- moisture of ground (range 4-40\%, at temperature from 0 before $+500 \mathrm{C}$ )

- a temperature of the air (the range from -50 before $+500 \mathrm{C}$ ):

- a temperature of ground (the range from -25 before $+500 \mathrm{C}$ );

- an photosynthetic to active radiation of the FAR (the range 0,38-0,70 maM) and other.

On description of the developer these sensors and functional converters "signalvoltage" is universal i.e. it for using suitable, as in automated measuring system, so and in separate discrete instrument.

Except sensor of moisture and the temperature of ground, sensor of the temperature and moisture of the air, sensor of the FAR automated agro meteorology point (AAP-1) includes many-server analog-digital converter united with RS at cable, or through radio link.
Computer provides processing and keeping agro meteorology to information. Base AAP-1 is completed 16 sensors.

In kit AAP-1 included sensors to moisture of ground for depths 10, 20, 30,..50 sm 15 piece), sensors of the temperature of the ground for depths 10, 20, 30,..50 sm 15 piece), sensors to moisture of the air (2 piece), sensors of the temperature of the air (2 piece) and sensors for measurement total and photosynthetic to active solar radiation (the FAR)- 2 piece.

Depending on request of the consumers amount channel can be increased before 64 and more for connection any amount sensor.

This will not disturb work AAP since speed his that allows "to interview" before 200300 sensors on length 1 seconds.

Alongside with foregoing methods, and exists the aerospace methods of the determination to moisture and temperature of ground.

On result called on by us analysis patent and research literature, denoted aerospace method of the checking the condition to surfaces of the land, in particular vegetation and ground gives the basis becomes firmly established that at development of the methods of such checking overland method and facility of the checking is conducted little attention. Designed in this direction of the models of the estimation of the condition of the distance of the plants and ground on satellite data, which are based on law optometrists mainly, thermodynamics and other physical law, will always require experimental check in overland condition.

A for this necessary reliable overland network of the checking condition plants and ground. 
However, herewith appear very complex and however, massive problems, connected with particularity aerospace and overland methods.

The first particularity: much differ the separate ability (the area incidence) object aerospace sensor and overland facility.

So if sensors companion land cover the area to terrestrial surface, which has a form cola by diameter from group of ten of the meter before several kilometers, that thermostats - weight method can characterize the wad of the land by diameter before 3-4 sm, in ditto time sensors VPG- $4 \mathrm{c}$ act in field, which has a form cola by diameter $60 \mathrm{sm}$ and more.

Relies on that if for cosmic sensor for separate ability to take the circle by diameter $10 \mathrm{ha}$, that volume of the sample for moisture to surfaces of ground for sensor companion will form 7,85*107 sm2, for VPG-4C - 2,826*103 sm2, but for TW-method - only $7,1 \mathrm{sm} 2$.

There is while one striking, way to take into account this particularity, this fascination on area of the number of the repetitions of the measurements instrument VPG-4C and, especially, determinations to moisture of ground TWmethod.

On the other hand, by means of aerospace methods it is impossible get sharing the temperature and moisture of ground on depth before 1-1,5 m.

In this case they while that no can change the overland facilities of the reception to information on moisture and temperature of the ground.

The second particularity: aerospace data, as a rule, do not comply with overland at time and space. The known that path companion often does not comply with location existing network hydrometeorology station and points, which lead the overland observations for condition of atmosphere, plants and ground. The periods agro meteorology observations also, chalice whole, do not comply with current of time of the passing artificial companion on location station and points.

To take into account this particularity there is two ways:

1) if well studied microclimate to particularities of the territory daily move agro meteorology of the factors, it is necessary to enter the corresponding to adjustments in given overland observations.

2) on route of the passing of the artificial companion beforehand to place the mobile agro meteorology points with increased the frequency of, than on hidro meteorology station period of the observations.

Such points allows not only more exactly fasten the overland observations to aerospace data, but can serve new, very efficient technical base for microclimate of the studies of the territory.

The third particularity: different physical principles prescribed in base overland and aerospace facilities of the measurement. In aerospace method and facility of the measurement are used several ways of the measurement parameter laying under surfaces of the land.

This registration reflected radiation in seen part spectrum, in infrared (IK) range and in radio-frequency radio diapason, as well as fixation natural gamma-radiations to surfaces of the land.

In instrument VPG-4C applying conduct meter way of the measurement to capacities of ground in variable electric current. TW-method is founded on weight 
drying and determination amount vaporized water.

Each of three above mention methods has their own advantage and defect. The advantage of the aerospace methods consists in therefore that they high-speed and cover the greater territory.

But they else have a defect in that that by means of their it is impossible get distribution an agro meteorology factors in deep cut before 1-1,5 $\mathrm{m}$ that that there is advantage of the overland facilities. The instrument VPG-4C has that superiority before TW-method that he characterizes the test of ground by volume 28 liters, but TW-method -only 22 milliliters.

TW-method except named above defect has its main advantage over VPG-4C and aerospace methods.

His advantage consists in that that he directs method and serves the facility graduation, both soils moisture meter, and aerospace facilities measurement to moisture of ground.

However attempt graduation cosmic sensors only by means of TW-method can be unsuccessful unless use VPG-4C as intermediate facility.

There by, necessary such correlation in use all that methods to possible was gets the most objective information on moisture of ground.

\section{Source of the literature}

Aliev Z.H. The methodical instructions on using of technologies pulsed drip irrigation in condition Azerbaijan. Azerbaijan Engineering - Building University, 1999, 39 p.
Alekseev A.S., Dementiev V.N. and others - In kn. : Cosmic methods of the study nature ambiences. Novosibirsk. 1983. p. 115-125.

Werigo S.A. Methodic scheduling the forecast spare productive moisture and estimation moisture provided corn cultures. - In kn.: Collection of the methodical instructions on analysis and estimation agro meteorology condition. L.GIDROMETIZDAT, 1977, p. 143-164.

Destination flexing: quantitative approach/ translation with engl., under red. The Member of the cortexes. AN USSR. A.S.Alekseeva.-M.:Depths, 1983.

Stepanov V.N. The biological categorization of the agricultural plants field culture. - Pub. TSHA, \# 2, 1957, p. 529.

Fedoseev A.P. "Aeromechanics and weather". - L:Hidrometizdat, 1976, 240 p.

Dmitrenko V.P., Grushka I.G. The meter parameter soil VPG-4C. Patent. 27798. The Ukraine's. VUG NPC SMSI ZPP. From 04.03.2004.

The complex methods of the processing aerospace photo information for revealing the particularities of the construction terrestrial cortex./A.L.Yashin, L.Zyatykova, V.N.sharapov and others- in kn.: Cosmic methods of the study of the natural ambience. Novosibirsk, 1983, 11$24 \mathrm{p}$. 
Use the remote methods for separation of the perspective areas within weakly study by territory/ V.Y. Eromenko, A.I. Prokopenko.- In kn.- Geological structures Siberia and their ore-bearing. Tomsk, TTU, 1983, @.2.p. 124-133

Loren E.N. Automatization and optimization.- M.: the World, 1975 